\title{
Helmet Detection on Two-Wheelers and Number Plate Recognition using Image Processing
}

\author{
C. Nikhita, Chaithanya S., Deeksha P., Shyma Zaidi
}

\begin{abstract}
There has been a sudden increase in motorcycle accidents over the years. The helmet is a safety equipment that protects the motorcyclists, however many riders (i.e. students) don't use it and the results could be fatal. This paper holds an agenda to propose a system for detection of motorcyclists without helmet. The proposed idea first detects motorcycle riders using surveillance video using background subtraction and object segmentation methods. We have also used techniques that involve human face detection using image processing. This includes face detection using Haar like feature technique and circular Hough transform method that helps in detecting any circular object hence detecting the helmet. This approach works in the near real time mode with significantly low false alarms.
\end{abstract}

Keywords: Helmet Detection, Number Plate Recognition, Image Processing, Two Wheelers.

\section{INTRODUCTION}

Motorcycle is a very popular mode of transportation among students in almost every country. However, it comes with a high risk of accidents. To reduce the involved risk, it is ideal for motorcyclists to use helmet [1-8]. Considering this, the government has made it a punishable offence to ride a motorcycle without the helmet [9]. Currently video surveillance based methods are being used $[6,9]$. These are quite passive and infeasible as humans are involved. Automation of this process is highly desirable for reliable and robust monitoring of these violations in college premises. Considering the desired properties and challenges, we propose an idea for automatic detection of motorcyclists without helmet, using input from existing surveillance cameras that work in real time. So, the solution for detecting violators using the existing equipment makes it cost-effective.

The rest of the paper is divided as follows:

Part II: The overview of the proposed approach.

Part III: Details of the proposed approach.

Part IV: Discussion

Part V: Conclusion

\section{OVERVIEW OF THE APPROACH}

Revised Version Manuscript Received on August 19, 2019.

C. Nikhita,Department of Electronics and Communication Engineering, Vidyavardhaka College of Engineering, Mysuru, Karnataka 570002 India.(email:mailnikhita4@gmail.com)

Chaithanya S, Department of Electronics and Communication Engineering, Vidyavardhaka College of Engineering, Mysuru, Karnataka 570002, India.(emailchaithanya.salanke@gmail.com)

Deeksha P, Department of Electronics and Communication Engineering, Vidyavardhaka College of Engineering, Mysuru, Karnataka 570002, India. (email:deeksha.prakash31@gmail.com)

Shyma Zaidi, Department of Electronics and Communication Engineering, Vidyavardhaka College of Engineering, Mysuru, Karnataka 570002, India.(email:shymazaidi@vvce.ac.in)
Detection of two-wheeler riders without helmet, to the college, requires effective automatic surveillance system that involves (i) surveillance cameras to capture the rider and the number plate. (ii) Haar like feature to detect the face of the rider. (iii) Circular Hough transform method to determine if the helmet is worn by the rider. If worn, it identifies if it is half or full helmet.

The entire information processed is stored in the database. If a rider fails to obey the rule more than once, a fine notice is sent through a message and an email to the rider with the help of Ubidots (IOT Platform).

For smooth and effective implementation of the procedure, the Haar like feature and the circular Hough transform must work hand in hand. The failure of one can result in disorganized execution. For instance, the Hough transform may confuse any other similar looking objects (circular) with a helmet, if the Haar feature is unsuccessful in detecting the face.

\section{DETAILS OF THE PROPOSED APPROACH}

The details of the approach are discussed in this section. As mentioned earlier it works in various phases. The first Phase detects the rider using surveillance camera captures.

In the second phase the head of the rider is located and identified if the helmet is worn.

The reading of the characters from name plate and storing the data, happens in the next phase. In the final phase, offender are automatically sent fine notice through emails and messages using Ubidots.

In order to maintain high accuracy and reduce false predictions, the procedure from the consecutive phases is consolidated.

Face detection using Haar like feature:

In this the most relevant features as far as humans are concerned such as the eyes (left eye, right eye), nose, lips, forehead and eyebrows are identified. Which can be done using the Haar like feature [10]. It is a sequence of rescaled square shaped functions, very similar to Fourier analysis. Haar feature can be assigned to every single important feature on the human face. For example, the edge features and the line features as shown in Fig. 1(a) and (b) respectively.

The edge feature is going to detect the edges, like the eyebrows contain darker pixels and the forehead contains lighter pixels, hence that region forms a horizontal edge feature as shown in Fig. 1(c). Similarly, the mouth region

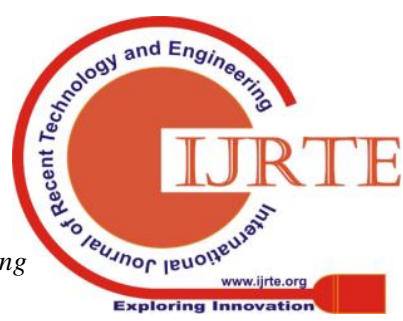


forms a line feature as, the lips have dark pixels and teeth have light pixels. Coming to the nose, the top part of the nose has light pixels and either sides of the nose have dark pixels (black-white-black).

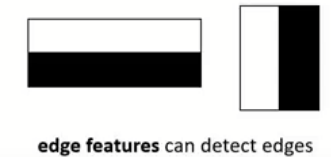

edge features can detect edges quite effectively

(a)

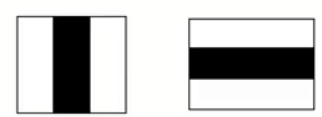

line features can detect lines quite effectively

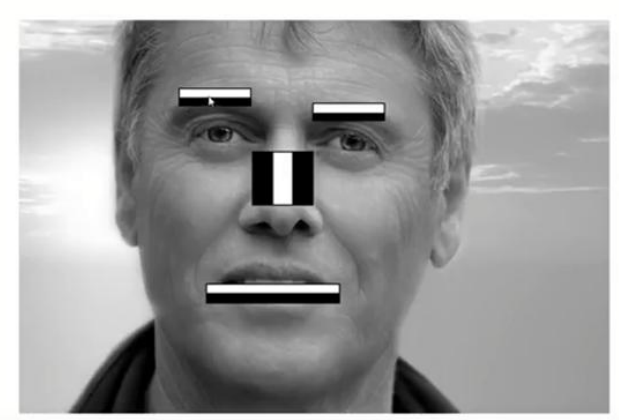

Figure 1: (a) Edge features, (b) Line features, (c) The most relevant features on the human face can be represented with Haar-Features. (Image Courtesy [11])

Hence, Haar feature is the most effective method to detect the most relevant features.

Concreate computation: Assign pixel intensities to every single pixel. In greyscale images, the range is from $0-255$. In an ideal Haar feature, 0 is white and 1 is black. However in real life scenarios, these values are not 0 's and 1 's excusively, since one is dealing with grescale images. The images shown in Fig.2 (a) and (b) demonstrate the pixel ranges in the ideal case and the real life values of Haar-features.

\begin{tabular}{|l|l|ll|}
\hline 0 & 0 & 1 & 1 \\
\cline { 1 - 2 } 0 & 0 & 1 & 1 \\
\cline { 1 - 2 } 0 & 0 & 1 & 1 \\
\cline { 1 - 2 } 0 & 0 & 1 & 1 \\
\hline
\end{tabular}

(a)

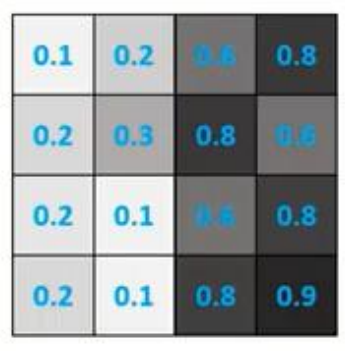

(b)
Figure 2(a) Ideal Haar features, (b) Real values of Haar-feature detected on an image. (Image Courtesy [11])

Viola-Jones algorithm can be used to compare how close the real scenario is to the ideal case.

1) Sum up the white pixel intensities and calculate the average.

2) Sum up the dark pixel intensities and calculate the average.

3) Delta $(\Delta)$ which is the difference between white and dark pixels is given by the following formula.

$$
\Delta=\text { dark }- \text { white }=\frac{1}{n} \sum_{\text {dark }}^{n} I(x)-\frac{1}{n} \sum_{\text {white }}^{n} I(x)
$$

$\Delta$ for ideal Haar-feature is 1

$\Delta$ for the real image: $0.74-0.18=0.56$

The closer the value to 1 , the more likely a Haar-feature is identified.

$\rightarrow$ Circular Hough method:
The principle of Hough transform can be applied to any shape. Here it is applied to a circle [12].

1) For this, the equation of the circle is defined which is given below.

$$
\left(x-x_{0}\right)^{2}+\left(y-y_{0}\right)^{2}-r^{2}=0
$$

2) After that a $3 \mathrm{D}$ accumulator array $\mathrm{A}$ is constructed (there are 3 unknowns). The dimensions here are $\mathrm{x} 0, \mathrm{y} 0$ and r0.

3) Fix one of the parameters and loop for the others

4) Increment corresponding entry in A.

5) Find the local maxima in A.

6) A gradient information (tangent direction) can be used at the edge point.

7) Compute $\mathrm{x} 0$, y0 given $\mathrm{x}, \mathrm{y}, \mathrm{r}$.

The circular Hough transform is subsequently applied in the procedure. It is applied in the surveillance cameras in the first phase to identify the helmets. The main limitation of this is the use of geometric resources to identify the helmet. In some cases the human head can be mistaken for a helmet due to the similar shape.

$\rightarrow$ Number plate detection using image processing:

This is the most prominent part of the approach.

The reference block diagram shown represents the process of character reading and character recognition of the number plate.

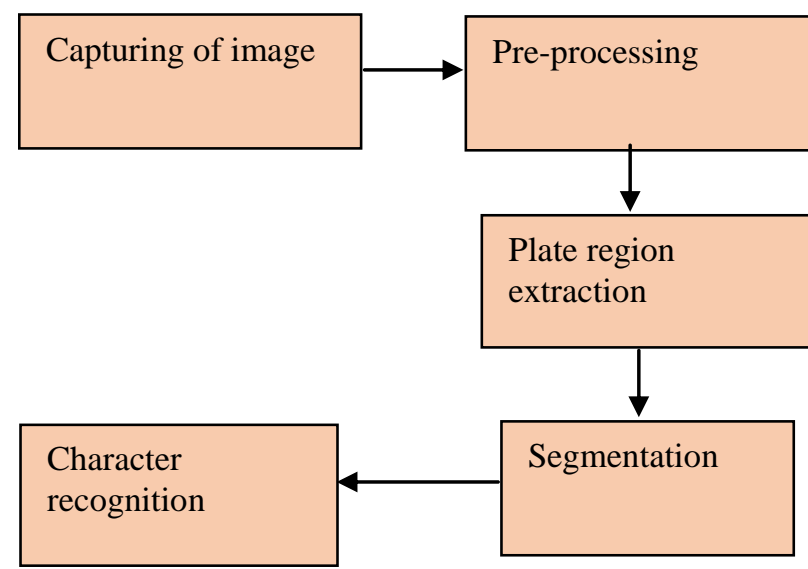

Figure 3: Block diagram to represent the process for character reading and character recognition of the number plate.

\section{Capturing of image:}

The first step is to capture the image of the number plate using surveillance camera. The image is then stored as colour JPEG format.

\section{Pre-processing:}

Noises are present in the image, when it is acquired. These noises should be removed from images using techniques like Gray processing

Median filtering 


\section{Plate region extraction:}

After pre-processing, the region where number plate is present i.e. characters need to be extracted.

\section{Segmentation of character in extracted plate:}

In this step, the output of the extracted number plate is obtained using labelling components, and then separate each character and split each character in number plate image by using split and also find length of number plate.

\section{Character recognition:}

The characters are segmented and recognised by comparing it to the database. This is done by comparing each value of the database with the character. If search matches, then the value is converted into string and display the output.

\section{Principle:}

The basic principle involved in this paper for number plate detection is digital image processing with the use of computer algorithms to create, process, communicate and display digital images.

They can be used to

Convert signals from an image sensor into digital images

Improve clarity, and remove noise

Extract the size, scale or number of objects.

\section{Description:}

After the pre-processing of the image and extraction, compare it with alpha numeric code in order to get image data converted into raw data. Hence this sequence of data is then compared with predefined database in order to get the details and know how many times the person has violated the rules and the person can be notified using Ubidots.

\section{DISCUSSION\& RESULTS}

This paper comes about the detection of a violator entering college without helmet. Here we propose a computer system that divides the program in detecting the number plate of violator's bike through moving the objects segmentation, moving objects classification and detecting helmet. This proposed approach has two parts one part is, it culminates the information of the image with human face detection algorithm. The other part works for helmet detection by circle Hough transform. The possible improvements can be that this technique can be extended for military purpose i.e. to keep a check on the number plates of the vehicles moving in restricted areas and can also be used to detect motorcyclists with triple riding.

\section{CONCLUSION}

In this paper, we propose a framework for detecting helmet who ride bike without using helmet. This framework can be done by noting down the number plates of violators in college. And this framework will also help us to detect the violators entering college without helmet in odd timings. We aim to apply some deep learning for improving this technique for overall accuracy of the system. These scenarios can be automatically adapted in real-time. The proposed system also obtained satisfactory hit rates in college. This system can be extended to the detection of more than one violator. An additional feature included is number plate detection where captured image of number plate is compared with database which helps in maintaining records of that number plate. The person will be notified for his violation of rules by sending a text messages or email.

\section{ACKNOWLEDGEMENT}

The authors express gratitude towards the assistance provided by Dr. Nikesh V V, Accendere Knowledge Management Services Pvt Ltd for the expert advice.

\section{REFERENCES}

1. R. Romuere and K. R. T. Aires, "Helmet Detection on Motorcyclists Using Image Descriptors and Classifiers," no. April 2017, 2014.

2. R. Waranusast and N. Bundon, "Machine Vision Techniques for Motorcycle Safety Helmet Detection," no. November 2013, 2016.

3. K. Dahiya, D. Singh, and C. K. Mohan, "Automatic Detection of Bike-riders without Helmet using Surveillance Videos in Real-time," no. December 2017, 2016.

4. S. A. Ghonge and J. B. Sanghavi, "Smart Surveillance System for Automatic Detection of License Plate Number of International Journal of Computer Sciences and Engineering Open Access Smart Surveillance System for Automatic Detection of License Plate Number of Motorcyclists without Helmet," no. January, 2018.

5. P. Doungmala, "Half and Full Helmet Wearing Detection in Thai- land using Haar Like Feature and Circle Hough Transform on Image Processing," 2016 IEEE Int. Conf Comput. Inf. Technol., pp. 611-614, 2016.

6. B. Duan, W. Liu, P. Fu, C. Yang, X. Wen, and H. Yuan, "Real-Time On-Road Vehicle and Motorcycle Detection Using a Single Camera," 2009 IEEE Int. Conf. Ind. Technol., pp. 1-6.

7. G. Sasikala, K. Padol, A. A. Katekar, and S Dhanasekaran, "Safeguarding of Motorcyclists Through Helmet Recognition," 2015 Int. Conf. Smart Technol. Manag. Comput. Commun. Control. Energy Mater., no. May, pp. 609-612, 2015.

8. Abhijeet S. Talaulikar, Sanjay Sanathanan, and Chirag N. Modi, "An enhanced approach for detecting helmet on motorcyclists using Image processing and Machine Learning techniques," Advanced Computing and Communication Technologies, Proceedings of the $11^{\text {th }}$ ICACCT, 109, 2018

9. $\mathrm{P}$ Wonghabut, J Kumphong, $\mathrm{T}$ Satiennam, $\mathrm{R}$ Ung-arunyawee, and WLeelapatra, "Automatic helmet-wearing detection for law enforcement using CCTV cameras Automatic helmet-wearing detection for law enforcement using CCTV cameras,'IOP Conf. Ser.: Earth Environ. Sci. 143, 012063, 2018.

10. https://en.wikipedia.org/wiki/Haar-like_feature

11. https://www.youtube.com/watch?v=F5rysk51txQ

12. https://en.wikipedia.org/wiki/Circle_Hough_Transform 